\title{
Amyloidosis of the Breast: Three Different and Unusual Presentations of a Rare Entity
}

\author{
Laura Herrero $^{a}$ Dolores Naranjo-Hans ${ }^{a}$ Manel Soléa Gorane Santamaría ${ }^{b}$ \\ Xavier Bargallób ${ }^{b}$ Martín Velasco $^{\text {b }}$ Pedro Luis Fernández ${ }^{a}$ \\ Departments of a Pathology and ${ }^{b}$ Radiology, Hospital Clinic, University of Barcelona, Barcelona, Spain
}

\section{Established Facts}

- Amyloidosis of the breast is a rare heterogeneous entity.

- It is usually of the AL type.

- There are no specific clinical or radiological features for breast amyloidosis.

\section{Novel Insights}

- Breast amyloidosis may be associated with CREST syndrome (calcinosis, Raynaud syndrome, esophageal dysmotility, sclerodactyly and telangiectasia).

- We report the first case described in the literature showing transthyretin deposits of amyloid in the breast that were associated with carcinoma.

\section{Key Words}

Breast · Amyloidosis · Crest syndrome · Transthyretin

\begin{abstract}
Background: Amyloidosis involving the breast is a rare finding and it may present as a solitary mass called 'amyloid tumor'. According to the largest case series, the amyloid deposits are usually of the AL type (commonly k light chain). Methods: We report 3 cases diagnosed at our institution in the period from 2000 to 2015. Radiological, histological and im-
\end{abstract}

munohistochemical studies were performed. Results and Conclusions: Together with a case presenting in a patient with multiple myeloma, we describe 2 unique presentations including 1 associated with CREST syndrome in a patient with a previous history of breast carcinoma and another, also associated with cancer, with transthyretin deposits in a woman with a TTR gene mutation and a family history of familial amyloidotic polyneuropathy. These cases are an example of the vast heterogeneity of this disorder regarding its clinical presentation, the type of amyloid deposits and other diseases associated with breast amyloidosis.

(c) 2015 S. Karger AG, Base

\section{KARGER 125}

(C) 2015 S. Karger AG, Basel

$1015-2008 / 15 / 0826-0264 \$ 39.50 / 0$

E-Mail karger@karger.com

www.karger.com/pat
Pedro Luis Fernández, MD, PhD

Department of Pathology, Hospital Clinic, University of Barcelona C/Villarroel 170

ES-08036 Barcelona (Spain)

E-Mail plfernan@ clinic.ub.es 


\section{Introduction}

Amyloidosis is a disorder characterized by interstitial deposits of different protein materials in a $\beta$-pleated sheet architecture. It may be clinically unexpected, and ultimately it is the pathologist who recognizes the deposits histologically in a hematoxylin and eosin (HE) stain as amorphous, homogeneous, eosinophilic material. The condition may be systemic, i.e. affecting several organs and systems, or else localized to one organ. More than 31 amyloid proteins have been described, the most common being AL protein, containing light-chain immunoglobulin, AA protein, which derives from a precursor protein of the liver and $A \beta$ amyloid, which is part of the brain plaques in Alzheimer's disease. Other proteins that have been identified in association with amyloidosis are transthyretin (TTR) in senile systemic amyloidosis, a mutated TTR form in familial amyloid polyneuropathy and $\beta_{2}$ microglobulin in patients with long-term hemodialysis, among other diseases.

Despite being a very rare condition, a series of 40 cases from the Mayo Clinic [1] concluded that amyloidosis of the breast is commonly of the AL type (usually $\kappa$ light chain); in half of the patients it was part of a systemic disease and in 55\%, it was associated with a hematologic malignancy in the breast (usually MALT lymphoma). None of their patients had invasive carcinoma of the breast.

We describe 3 cases of breast amyloidosis with different and unusual clinical and radiological presentations over a 15-year period from our hospital records, 2 of which were associated with carcinoma.

\section{Case Reports}

Three cases of breast amyloidosis were retrieved after reviewing our archives for the period from 2000 to 2015. Congo red staining was performed in all cases, and it was examined under polarized light to detect the typical apple-green birefringence. Amyloid $A$, TTR and $\kappa$ and $\lambda$ light chains were immunohistochemically analyzed in order to typify the deposits of amyloid.

\section{Case 1}

In a screening mammography, a 78-year-old woman with a clinical history of CREST syndrome (calcinosis, Raynaud syndrome, esophageal dysmotility, sclerodactyly and telangiectasia) presented with a mass in the retroareolar region of the right breast with a retracted nipple. Cytology was performed with a diagnosis of atypical cells consistent with ductal carcinoma. Right mastectomy and axillary lymphadenectomy were performed. Light microscopy showed mixed patterns of invasive ductal, papillary and lobular carcinoma. Metastases were found in 4 of 12 regional lymph nodes. Treatment with chemotherapy, radiotherapy and tamoxifen was decided on. One year later, she presented with a $5-\mathrm{cm}$ mobile mass in the contralateral breast; a mammography showed a retroareolar mass with coarse calcifications (fig. 1a). The resection specimen showed a fibrous area with very hard, apparently calcified foci (fig. 1b). Microscopy showed stromal deposits of hyaline material around the ductal structures and small vessels (fig. 1c) which had green birefringence under polarization with Congo red staining (fig. 1d), that was consistent with amyloid deposits. Areas of extensive osseous metaplasia and a foreign-body granulomatous reaction were also present in nearby zones (fig. 1e). The lesion was diagnosed as an 'amyloid tumor'. The amyloid deposits were immunoreactive for $\kappa$ and $\lambda$ light chains (fig. 1f). Review of the slides from the prior surgery of the right breast showed no amyloid deposits. The patient died several months later due to complications of CREST syndrome.

\section{Case 2}

A 54-year-old woman with a diagnosis of quiescent multiple myeloma in 2010 with lesions in the eyelids was diagnosed as having AL type amyloidosis with $\lambda$ light chain deposits. She underwent screening mammography 2 years later. The mammogram showed a diffuse increase of adipose tissue density in both breasts, consistent with amyloidosis. Biopsy confirmed deposits of amyloid (fig. 2a) which showed $\lambda$ light chain restriction on immunohistochemistry (fig. 2b, c). She received an autologous bone marrow transplantation in 2013. No significant changes have been observed in the breast amyloidosis since then.

Case 3

A 51-year-old woman with a TTR Val30Met mutation detected at screening in 2001 presented with TTR-related familial amyloidotic polyneuropathy 7 years later and underwent a liver transplantation. Annual screening mammographies only showed fibrocystic disease. In 2013, the patient was referred to our hospital for further evaluation of a ductal carcinoma in situ grade 3 with comedonecrosis that had been detected by needle biopsy performed at another institution. A mammography showed a group of microcalcifications involving a $4-\mathrm{cm}$ area in the confluence of the lower quadrants of the right breast. The ultrasonography study showed a solid, polylobulated mass of heterogeneous content that measured $4 \mathrm{~cm}$ (fig. $2 \mathrm{~d}$ ). The needle biopsy showed periductal hyaline deposits of amyloid with no evidence of neoplastic process. The right breast and sentinel axillary lymph nodes were resected. The resection specimen showed a Bloom-Richardson grade 3 microinvasive ductal carcinoma with extensive ductal carcinoma in situ. The deposits of amyloid were reactive for TTR (fig. 2e) and located near the carcinoma in situ component and normal ducts (fig. $2 \mathrm{f}$, $\mathrm{g}$ ), and $\kappa$ and $\lambda$ light chains were detected without evidence of restriction. The axillary sentinel lymph nodes were negative for metastatic carcinoma. She underwent treatment with trastuzumab and aromatase inhibitors after surgery.

\section{Discussion}

Amyloidosis involvement in the breast is uncommon; it was first reported by Fernandez and Hernandez [2] in 1973. Most patients are women but it may also affect men. 

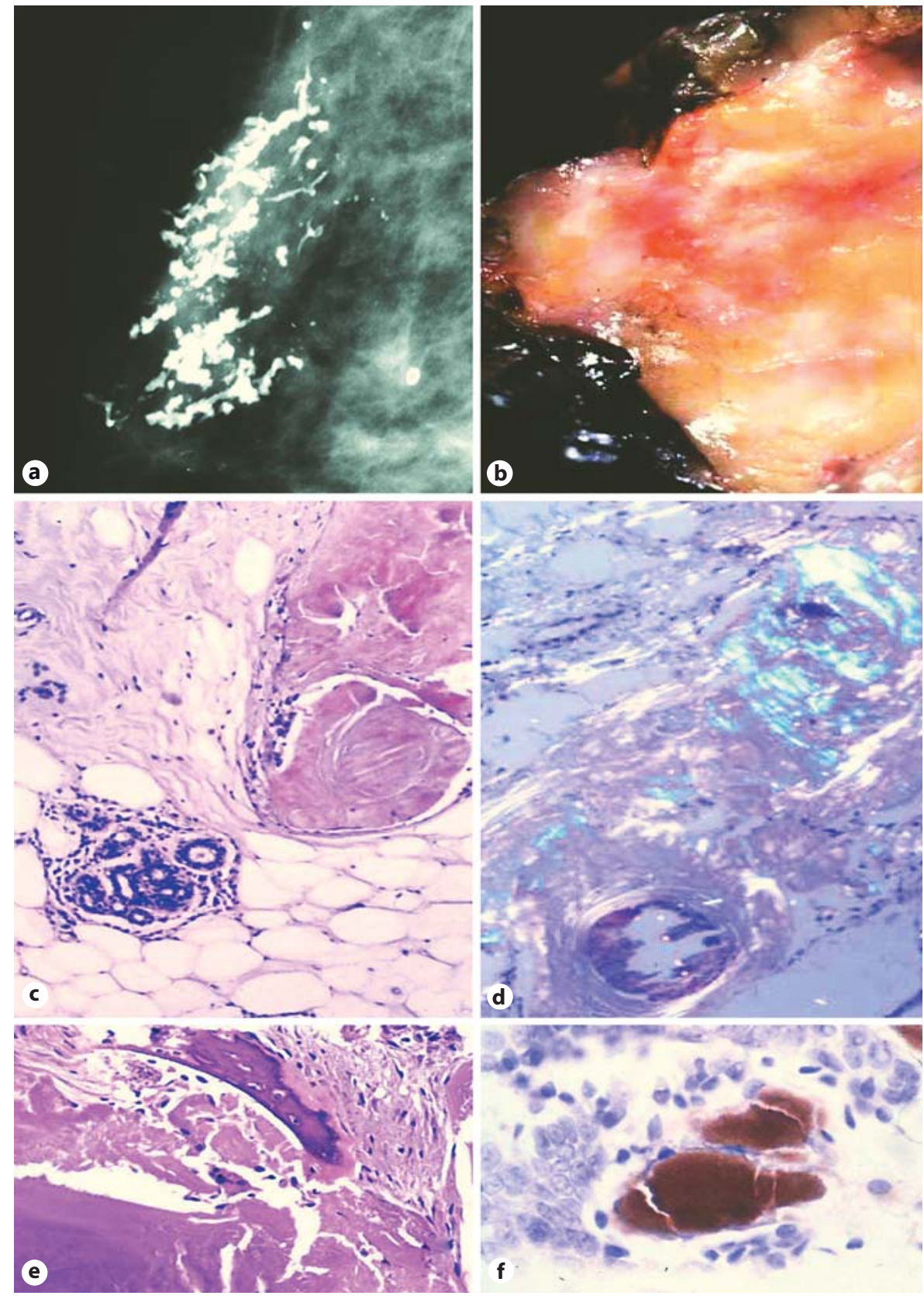

Fig. 1. Case 1. a Mammography of the left breast showing coarse retroareolar calcifications. b Surgical specimen showing whitish hard and brittle areas. c Stromal periductal hyaline deposits forming nodular aggregates. HE. d Green birefringence under polarized light. Congo red. e Osseus metaplasia near amyloid deposits. HE. f Immunostaining for $\lambda$ light chain.

The age at diagnosis ranges from 35 to 86 years. It is usually unilateral, but bilateral involvement has also been described $[1,3]$. The clinical presentation is usually a painless, solitary mass which may show calcifications on mammography, raising the suspicion of breast cancer. Systemic amyloidosis is more common than deposits limited to the breast. Thus, this entity was only suspected radiologically in case 2 as this patient had multiple myeloma. Bilateral breast involvement by amyloidosis was demonstrated in this case. In the other two cases, the clinical suspicion was breast cancer, but invasive carcinoma was histologically confirmed only in case 3 . In case 1 , only an amyloid tumor was diagnosed, although the patient had presented with cancer in the contralateral breast the year before. A case of a woman with an amyloid tumor 2 years after having had an invasive ductal carcinoma was reported previously [4]. In that case, the deposits occurred in the same breast, i.e. mimicking recurrence 
Fig. 2. a-c Case 2. a Deposits of amyloid in adipose tissue. HE. $\mathbf{b}$ Immunostaining for $\kappa$ light chain. $\mathbf{c} \lambda$ light chain restriction. DAB. d-g Case 3. d Ultrasonography showing a solid polylobulated mass of heterogeneous content measuring $4 \mathrm{~cm}$. e Immunostaining showing amyloid deposits positive for TTR. f Amyloid deposits close to ductal carcinoma in situ. Congo red staining. $\mathbf{g}$ The same sample (as in f) shows apple-green birefringence under polarized light.

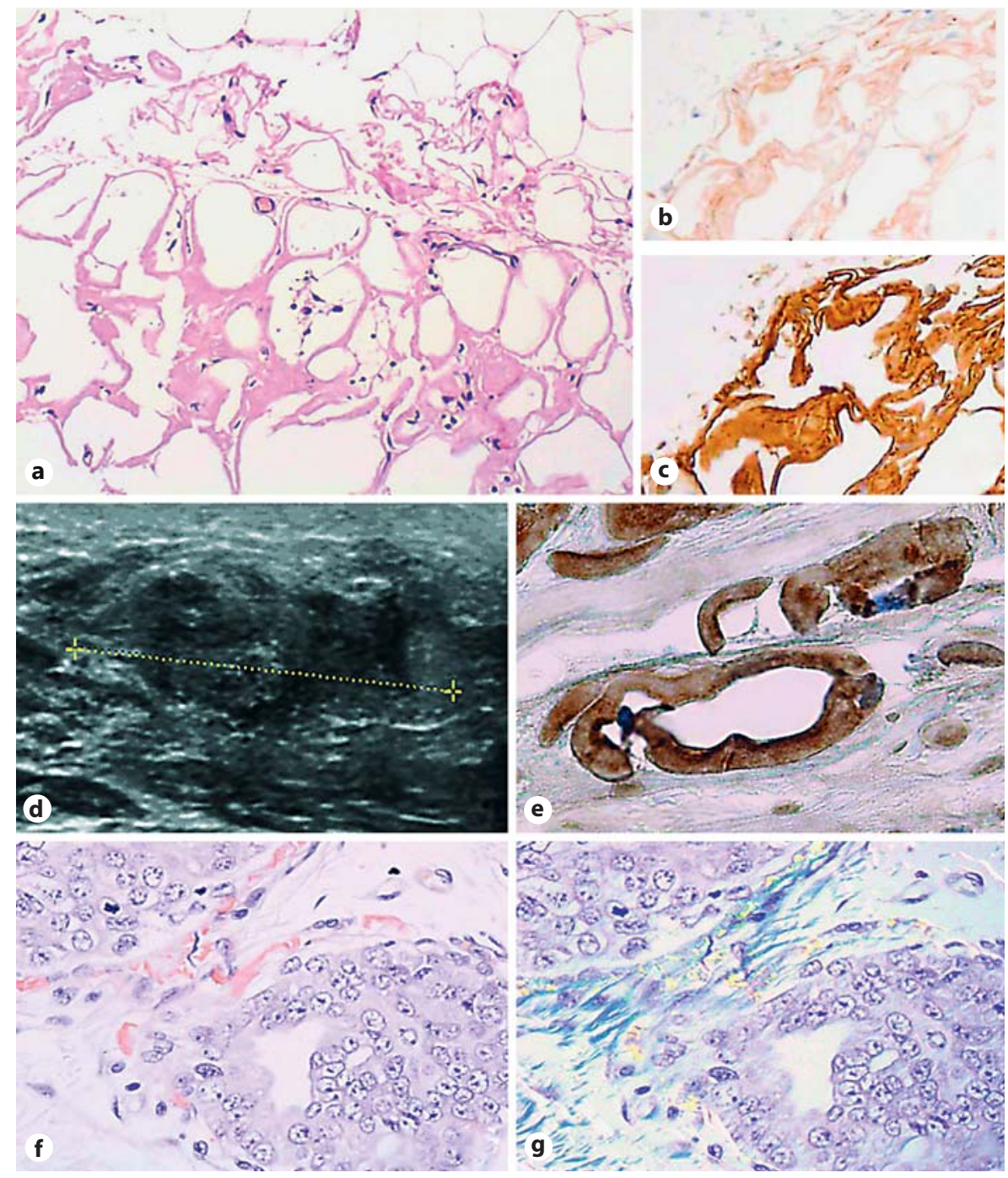

whereas in our patient, the amyloid tumor was in the contralateral breast. Generalized amyloid disease was not investigated in our patient because she died shortly thereafter.

Some authors have described breast amyloidosis as a distinct lesion while others have reported it in association with nonmalignant and malignant entities. It has been related to breast cancer showing different histologies (medullary type carcinoma, tubular carcinoma, invasive ductal carcinoma, invasive lobular carcinoma, pleomorphic lobular carcinoma and carcinoma in situ) [1, 3, 5-8]. In some cases, the radiologic presentation consisted of coarse calcifications detected on a screening mammography $[5,9]$. Therefore, a breast carcinoma has to be ruled out. In the series by Charlot et al. [5] only one patient showed coex- isting invasive ductal carcinoma. Our case 3 had a $4-\mathrm{mm}$ invasive ductal carcinoma with deposits of amyloid in proximity to the extensive ductal carcinoma in situ component. Amyloidosis has also been described as being associated with hematologic disorders of the breast such as MALT lymphoma, chronic lymphoid leukemia, marginal zone lymphoma and diffuse large B cell lymphoma [1, 3, $10,11]$. The group of nonmalignant diseases related to breast amyloidosis includes rheumatoid arthritis $[3,12]$. Patient number 1 had CREST syndrome, which could have had a role in the development of the amyloidosis. This is, to our knowledge, the first report of an association between amyloidosis and CREST syndrome.

According to the literature, breast amyloidosis is commonly of the AL type, usually $\kappa$ [1]. Most patients with 
AL-type breast amyloidosis have no associated multiple myeloma or lymphoma; therefore, it is currently thought that this variant of localized breast amyloidosis is derived from local plasma cells secreting immunoglobulins [13]. In cases 1 and 2, the deposits of amyloid were of the AL type; the first showed no light chain restriction and the second was $\lambda$ light chain immunoreactive.

TTR amyloid deposits in the breast have rarely been reported. According to our knowledge, only 3 cases of female patients with the Val30Met mutation and symptoms of familial amyloidotic polyneuropathy showing deposits of amyloid in the breast have been published [14]. TTR amyloid was also reported as a finding in the breast biopsy of an 80-year-old woman without a TTR gene mutation [15]. Interestingly, our third case is the first described in the literature showing TTR deposits of amyloid in the breast associated with carcinoma in a patient with a TTR gene mutation and familial amyloidotic polyneuropathy.
We conclude that breast amyloidosis is a heterogeneous and very infrequent disorder. However, when a diagnosis is made, it is mandatory to rule out a hematologic disorder and systemic involvement. In contrast, abnormalities on breast imaging such as coarse calcifications or an increase of density of the adipose component of the breast in patients with systemic pathology such as myeloma and autoimmune conditions must include mammary amyloidosis in the differential diagnosis.

\section{Acknowledgements}

This work was supported by Generalitat de Catalunya Suport Grups de Recerca AGAUR 2014-SGR-795, Red Temática de Investigación Cooperativa en Cáncer (RTICC) grant RD12/0036/0036 and Xarxa de Bancs de Tumors de Catalunya (XBTC).

\section{References}

1 Said SM, Reynolds C, Jimenez RE, Chen B, Vrana JA, Theis JD, Dogan A, Shah SS: Amyloidosis of the breast: predominantly AL type and over half have concurrent breast hematologic disorders. Mod Pathol 2013;26:232238.

2 Fernandez BB, Hernandez FJ: Amyloid tumor of the breast. Arch Pathol 1973;95:102-105.

3 Röcken C, Kronsbein H, Sletten K, Roessner A, Bässler R: Amyloidosis of the breast. Virchows Arch 2002;440:527-535.

4 Toohey JM, Ismail K, Lonergan D, Lewis CR: Amyloidosis of the breast mimicking recurrence in a previously treated early breast cancer. Australas Radiol 2007;51:594-596.

5 Charlot M, Seldin DC, O'hara C, Skinner M, Sanchorawala V: Localized amyloidosis of the breast: a case series. Amyloid 2011;18:72-75.

6 Chiang D, Lee M, Germaine P, Liao L: Amyloidosis of the breast with multicentric DCIS and pleomorphic invasive lobular carcinoma in a patient with underlying extranodal Castleman's disease. Case Rep Radiol 2013;2013: 190856.
7 Sabate JM, Clotet M, Torrubia S, Guerrero R, Pineda R, Lerma E, Capdevila E: Localized amyloidosis of the breast associated with invasive lobular carcinoma. Br J Radiol 2008; 81:e252-e254.

8 Munson-Bernardi BD, DePersia LA: Amyloidosis of the breast coexisting with ductal carcinoma in situ. AJR Am J Roentgenol 2006; 186:54-55.

9 Fu K, Bassett LW: Mammographic findings of diffuse amyloidosis and carcinoma of the breast. AJR Am J Roentgenol 2001;177:901902.

10 Lui PC, Pang LM, Hlaing TT, Tse GM: Tumorous amyloidosis of the breast associated with disseminated malignant lymphoma. J Clin Pathol 2004;57:334-335.

11 Kambouchner M, Godmer P, Guillevin L, Raphaël M, Droz D, Martin A: Low grade marginal zone B cell lymphoma of the breast associated with localised amyloidosis and corpora amylacea in a woman with long standing primary Sjögren's syndrome. J Clin Pathol 2003;56:74-77.
12 Tutar E, Onat AM, Aydin A, Kervancioğlu S, Buyukhatipoglu H, Inan G, Pehlivan Y: Amyloid tumor of the breast mimicking breast carcinoma. South Med J 2008;101:199-201.

13 Deolekar MV, Larsen J, Morris JA: Primary amyloid tumour of the breast: a case report. J Clin Pathol 2002;55:634-635.

14 Tokuda T, Takei Y, Takayama B, Hoshii Y, Ikeda S: Severe amyloid deposition in mammary glands of familial amyloid polyneuropathy patients. Amyloid 2007; 14:249-253.

15 Eriksson M, Butnner J, Todorov T, Yumlu S, Schonland S, Hegenbart U, Kristen AV, Dengler T, Lohse P, Helmke B, Schmidt H, Rocken C: Prevalence of germline mutations in the TTR gene in a consecutive series of surgical pathology specimens with ATTR amyloid. Am J Surg Pathol 2009;33:58-65. 